\title{
AVALIAÇÃO CLÍNICA E CITOLÓGICA DO TRATO RESPIRATÓRIO DE CAVALOS DA RAÇA QUARTO DE MILHA, APÓS O EXERCÍCIO
}

\author{
(Clinical and cytologic evaluation of respiratory tract from \\ quarter horses following exercise)
}

\author{
BIAVA, J.S. ${ }^{1}$; GONÇALVES, R.C. ${ }^{2}$; DORNBUSCH, P.T. ${ }^{3}$; MICHELOTTO JÚNIOR, P.V.; \\ BIONDO, A.W. ${ }^{4}$; CASSOU, F. ${ }^{5}$ ZANOTTO, G.M. ${ }^{6}$; TELLES, J.E.Q. ${ }^{7}$ \\ 1Pós-graduanda da Clínica Veterinária da FMVZ - Unesp / Botucatu - SP; \\ ${ }^{2}$ Docente da Clínica Veterinária da FMVZ - Unesp / Botucatu - SP; \\ ${ }^{3}$ Docente de Clínica e Cirurgia de Eqüinos - PUC - PR; \\ ${ }^{4}$ Docente Departamento de Medicina Veterinária da UFPR - PR; \\ ${ }^{5}$ Pós-graduanda de Medicina Veterinária da UFPR - PR; \\ ${ }^{6}$ Acadêmico de Medicina Veterinária da UFPR - PR; \\ ${ }^{7}$ Médico do Departamento de Patologia do Hospital de Clínicas / UFPR - PR.
}

RESUMO - O objetivo do presente estudo foi avaliar a citologia do lavado broncoalveolar (BAL) após o exercício de doze cavalos da raça Quarto de Milha, provenientes de Curitiba e Região Metropolitana. Após a infusão de $100 \mathrm{ml}$ de solução fisiológica com auxílio de endoscopia e cateter de silicone, foram recuperados acondicionados em torno de $30 \mathrm{~mL}$ de BAL por cavalo, analisados e submetidos à centrifugação. Lâminas de citocentrífuga foram obtidas e coradas pelas técnicas de Giemsa, Papanicolaou e Azul da Prússia. Nenhum dos cavalos apresentou epistaxe ou outra anormalidade ao exame clínico, nem sangramento pulmonar à endoscopia. A contagem de hemácias e leucócitos no BAL esteve dentro dos valores de referência encontrados na literatura, assim como os diferenciais

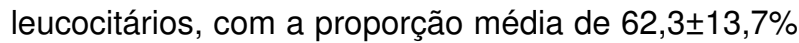
e $34,1 \pm 14,8 \%$ para macrófagos e linfócitos, respectivamente. No entanto, a presença de hemossiderófagos em diferentes percentuais, sugere que parte destes animais estejam sob hemorragia pulmonar incipiente, não detectada clinicamente. Concluiu-se, baseado no presente trabalho, que a avaliação das populações celulares com o uso da citocentrífuga em associação a colorações especiais pode fornecer ao clínico importantes informações adicionais acerca do status funcional do trato respiratório, em particular hemorragias subclínicas.

Palavras-chave: Citologia, Lavado Broncoalveolar, citocentrífuga, cavalo, Quarto de Milha

\footnotetext{
ABSTRACT - The aim of this study was to evaluate the bronchoalveolar lavage (BAL) cytology following exercise from twelve Quarter Horse breed animals from Curitiba City and surroundings. After infusion of $100 \mathrm{ml}$ of physiologic solution with endoscopy and silicon catheter, an average of $30 \mathrm{~mL}$ of BAL was recovery per horse, analized and submited to cytocentrifugation.
}

Cytocentrifuge slides were obtained and stained by Giemsa, Papanicolaou and Perl's Prussian Blue. None of the horses presented epistaxis or any other abnormality on the clinical examination, nor pulmonary bleeding by endoscopy. The erythrocyte and leukocyte in BAL counts were with in the normal range of literature, as well as the leukocyte differential, with a mean proportion of $62.3 \pm 13.7 \%$ and $34.1 \pm 14.8 \%$ for macrophages and lymphocytes, respectivelly. However, the presence of hemosiderophages in different percentages, suggested that part of the animals were under subclinical pulmonary hemorrhage, not clinically detected. Based on the present study, we concluded that the evaluation of cellular populations with the use of cytocentrifuge and special stains can provide to the clinician important additional information on functional status of respiratory tract, particularly hemorrhage.

Key-words: Cytology, BAL, cytocentrifuge, equine, Quarter Horse.

\section{Introdução}

O exame citológico das vias aéreas inferiores pode ser realizado através de aspirado transtraqueal e de lavados traqueal, traqueobrônquico e broncoalveolar (BAL) que auxiliam na avaliação das doenças do trato respiratório (FOGARTY, 1990; ERICKSON e POOLE, 2003). O BAL envolve a lavagem das vias aéreas inferiores, através de endoscópio ou cateter introduzidos em um brônquio (SWEENEY e BEECH, 1991; COWELL e TYLER 1992; HEWSON e VIEL, 2002). O BAL é a técnica mais sensível para avaliar o meio celular dentro de um segmento de brônquio, bronquíolo e alvéolos, sendo o método de seleção mais adequado para avaliar as vias aéreas distais (FORGARTY e BUCKLEY, 1991; CLARK et al., 1995; ERICKSON e POOLE, 2003). MAZAN e HOFFMAN (2003), referem 
Avaliação clínica e citológica do trato respiratório de cavalos da raça quarto de milha, após o exercício

em seu estudo uma significativa diferença entre o BAL de cavalos de corrida e cavalos de outros esportes, sendo que estes últimos apresentaram uma quantidade significativamente maior de neutrófilos, indicando uma maior cronicidade do processo inflamatório nestes animais.

A interpretação citológica varia com o método de colheita da amostra e a técnica de processamento utilizada (HEWSON e VIEL, 2002), assim como o tipo de líquido infundido, $\mathrm{pH}$, volume, diâmetro do material de coleta e o tempo de estocagem da amostra antes do processamento (MCGORUM et al., 1993; HEWSON e VIEL, 2002). A avaliação citológica é feita pela contagem diferencial de macrófagos, neutrófilos, linfócitos, eosinófilos, mastócitos e células cilíndricas do epitélio das vias aéreas (SWEENEY et al., 1992; CLARK et al., 1995; HEWSON e VIEL, 2002). A citologia pode atestar a hemorragia pulmonar, quando houver presença de macrófagos alveolares fagocitando eritrócitos ou com hemossiderina no seu citoplasma (COWELL e TYLER, 1992; OLIVER et al., 2003). Segundo MCKANE e SLOCOMBE (1999), são observados poucos hemossiderófagos nos primeiros três dias após a hemorragia pulmonar e grande quantidade de hemossiderófagos e mesmo de eritrócitos totalmente preservados ainda 14 dias após a hemorragia, caracterizando a dificuldade do sistema mucociliar e fagocitário em remover a hemorragia presente. Os autores observaram ainda pequeno número de células no lavado broncoalveolar realizado no $21^{\circ}$ dia após a hemorragia pulmonar. Além disso, pode-se detectar processos inflamatórios quando há aumento no número de neutrófilos e suspeitar de processos alérgicos, fúngicos ou reações a metazoários quando houver aumento de eosinófilos.

Para a interpretação citológica da amostra dois procedimentos devem ser realizados: uma lâmina direta, no momento da coleta, para a contagem total de células, e outra utilizando a citocentrífuga para a contagem diferencial das células. A lâmina preparada pela citocentrífuga é preferida pelos clínicos, pois muitas células são concentradas em uma área da lâmina facilitando assim a contagem diferencial em suas diferentes colorações (HEWSON e VIEL, 2002). Para maior acurácia na interpretação dos resultados, muitos citologistas preferem realizar a contagem diferencial de, no mínimo, 5 células por campo de visão no maior aumento ou 300 a 500 células por lâmina (MAZAN e HOFFMAN, 2003).

Apesar de vários estudos realizados em cavalos de corrida, pouco se sabe sobre a importância da hemorragia pulmonar induzida por exercício (EIPH) em animais de trabalho ou esporte. O objetivo do presente estudo foi de avaliar clinicamente e citologicamente o BAL de cavalos da raça Quarto de Milha após o exercício, com o uso da citocentrífuga e colorações especiais.

\section{Material e Método}

Foram utilizados 12 eqüinos da raça Quarto de Milha, 6 machos e 6 fêmeas, com média de 5,3 $\pm 3,0$ anos de idade e variando entre 3 a 14 anos, pesando $477 \pm 26$ $\mathrm{kg}$, clinicamente saudáveis, provenientes de Curitiba e Região Metropolitana, Estado do Paraná. Os cavalos eram mantidos em piquetes durante o dia e encocheirados durante a noite, alimentados com feno de alfafa, ração comercial e água ad libitum, além de vermifugados a intervalos de 60 dias. O regime de exercícios era de uma hora diária, seis dias na semana, incluindo a participação em campeonatos de tambor e baliza.

O exame clínico foi realizado em todos os animais 30 minutos após o exercício e previamente à endoscopia. Foram avaliados: a frequência respiratória, frequência cardíaca, estado de hidratação, coloração de mucosas, tempo de preenchimento capilar (TPC), linfonodos e atitude ao exame.

Os animais foram examinados visualmente através de endoscópio flexível (Colonoscópio CF 140L, Olympus Company, EUA), introduzido na narina em direção à traquéia e brônquios, com prévia sedação quando necessário. Foi utilizado um colonoscópio, marca Olympus, com 1,80 metros de comprimento e 12,80 $\mathrm{mm}$ de diâmetro externo com fonte de luz fria de 150 Watts. No trato respiratório superior e inferior, verificouse a presença de secreção nasal e epistaxe, a presença de secreção traqueal, alterações da bifurcação traqueal (carina), alterações nos brônquios principais e outros achados relevantes. A presença de hemorragia pulmonar induzida por exercício (EIPH) foi observada e, quando necessário, graduada de Grau 0 a Grau IV segundo escala de Eppinger (EPPINGER, 1990). Após a visualização das vias aéreas o endoscópio foi retirado e um cateter de silicone (Bivona, Smiths, Reino Unido) introduzido até um brônquio, sendo utilizada somente infusão de anestésico local (Lidocaína 1\%) para diminuir o reflexo de tosse.

Para a realização do lavado foram inoculados 100 $\mathrm{ml}$ de solução fisiológica comercial, o líquido introduzido sendo removido rapidamente por sucção, com seringa descartável de $60 \mathrm{ml}$ e devidamente identificado. Concluída a aspiração da amostra, a seringa foi desacoplada da sonda, o conteúdo homogeneizado e uma alíquota de $3 \mathrm{ml}$ foi separada para exame físicoquímico e citológico quantitativo do BAL. O restante do fluído foi colocado em uma caixa de isopor com gelo em temperatura de 0 a $4^{\circ} \mathrm{C}$ e encaminhada imediatamente ao Laboratório de Patologia do Hospital de Clínicas da Universidade Federal do Paraná $(\mathrm{HC} /$ UFPR), onde as amostras conservadas sob refrigeração foram centrifugadas em citocentrifuga (Revan Citocentrífuga 2000 D), durante 5 minutos. O botão celular formado na lâmina foi corado pelas técnicas de Giemsa, Papanicolau e Azul da Prússia, segundo metodologia padrão de confecção (KEEBLER e SOMKAK, 1993). Para a análise diferencial de células, 
BIAVA, J.S. et al.

foram contadas, quando possível, um total de 400 células, diferenciando-se as proporções de células epiteliais cilíndricas, neutrófilos, linfócitos, eosinófilos, mastócitos e macrófagos.

À análise descritiva das lâminas avaliou-se a celularidade, a presença de muco, hemácias, leucócitos e células, íntegras ou degeneradas. As lâminas foram processadas com o uso de citocentrífuga e todas analisadas para diferencial de células.

Os exames físico-químicos e citológicos quantitativos foram realizados no Laboratório de Patologia Clínica do Hospital Veterinário da UFPR, por observação da amostra e com o uso de tiras reagentes de urina (Uri-test, Inlab, São Paulo). O líquido foi avaliado quanto ao volume recuperado, aspecto, coloração, densidade específica, $\mathrm{pH}$, proteínas, glicose, sangue oculto e bilirrubina. Para o exame citológico quantitativo o líquido do BAL foi homogeneizado, diluído quando necessário, sendo as hemácias e células nucleadas totais contadas com o auxílio de câmara de Neubauer em microscópio óptico.

\section{Resultados}

Nenhum dos animais necessitou de prévia sedação para o exame clínico ou sondagem. Durante o exame físico constatou-se que a atitude, os linfonodos palpáveis, o estado de hidratação e a coloração de mucosas não apresentaram alterações detectáveis. Observou-se 21,1 \pm 6,7 movimentos respiratórios por minuto, 41,5 $\pm 9,5$ batimentos cardíacos por minuto e $2,1 \pm 0,3 \mathrm{seg}$. de tempo de preenchimento capilar. Nenhum dos animais apresentou epistaxe ao exame externo; apenas o cavalo 1 apresentou secreção mucosa nasal.

Não foi observado sangramento à endoscopia nos animais deste experimento. Havia presença de pequena quantidade de secreção assemelhando-se à saliva no terço médio da traquéia dos cavalos 4, 7, 10 e 12; no terço proximal, dos cavalos 2, 5 e 8; no terço distal, do cavalo 1 e em todas as porções da traquéia, do cavalo 3. Não foi observada a presença de secreção na traquéia dos animais 6, 9 e 11. Não foram observadas alterações da bifurcação traqueal (carina), alterações nos brônquios principais ou quaisquer outros achados relevantes como mostra a QUADRO 1.

Os lavados observados foram classificados como transparente e ligeiramente turvos. A fita reagente foi utilizada no BAL de 7 animais, sendo todos negativos para glicose e bilirrubina, além disso, apresentaram traços de proteínas, densidade específica média de $1,003 \pm 0,002$ e pH de 5,0 $\pm 0,0$. A tira reagente mostrou sangue oculto em 5 das 7 amostras avaliadas, com média de duas cruzes (101,4 \pm 137,3 hemácias/îL) .

Os valores de volume de infusão recuperados, contagem de hemácias, contagem de leucócitos e outras células, diferencial leucocitário e percentual de hemossiderófagos (contatos como parte do total de macrófagos) encontram-se resumidos na TABELA 1. A avaliação da composição dos grumos celulares foi realizada com o auxílio de lâminas coradas com Papanicolau, e estavam presentes nas lâminas dos cavalos 1, 5, 6, 8, 9, 10, 11, e 12. A avaliação e contagem de hemossiderófagos foi realizada com a lâmina corada pelo método de Azul da Prússia, sendo observada nos animais de números $1,4,5,9,10,11$ e 12, a proporção de $25 \%, 76 \%, 15 \%, 19 \%, 50 \%$, $40 \%$ e $11 \%$ do total de macrófagos, respectivamente.

\section{Discussão}

Neste estudo observou-se que a avaliação clínica não mostrou alterações relevantes, uma vez que todos os animais utilizados eram saudáveis. Nossos achados são semelhantes ao de BACCARIN (2005), que demonstrou que as freqüências cardíaca e respiratória podem estar elevadas logo após o exercício sem apresentar alterações durante o repouso, mesmo em cavalos com algum grau de hemorragia pulmonar.

Segundo os resultados obtidos, apenas três cavalos foram positivos na fita reagente para sangue oculto. Os dados obtidos pela fita de urinálise não foram sensíveis para detectar hemorragia pulmonar.

A utilização da técnica de lavado broncoalveolar (BAL) com auxílio de endoscopia é importante instrumento na avaliação citológica das vias respiratórias inferiores, permitindo diagnóstico mais preciso das afecções respiratórias em eqüinos. A grande vantagem do uso da endoscopia para a coleta do BAL é a visualização das estruturas das vias aéreas superiores e inferiores, definindo com isso, suas alterações anatômicas (ROY e LAVOIE, 2003). Além disso, podese estimar a severidade da EIPH avaliando a quantidade de sangue presente nas vias aéreas, pois a presença de epistaxe, ocorre somente em 0,25 a $13 \%$ dos cavalos de corrida acometidos de EIPH (ERICKSON e POOLE, 2003). Neste estudo, não foi observado nenhum grau de hemorragia pulmonar segundo a escala de EPPINGER (1990); entretanto, quando se utilizou o BAL, 58,3\% dos cavalos que estavam em exercício intenso durante o treinamento ou que haviam participado de provas nos últimos 21 dias, apresentaram citologicamente, alguns sinais de EIPH. Poucos são os estudos que utilizaram a avaliação de hemossiderófagos como parte da análise do BAL em cavalos. CLARK et al. (1995) observaram que os hemossiderófagos podem ser utilizados no diagnóstico de hemorragias subclínicas, uma vez que o sangramento não foi visível pela endoscopia. De acordo com BACCARIN (2005), o BAL é mais específico e sensível do que o exame endoscópico após exercício (Endoscópio = 1 a 2 horas após exercício; $B A L=1$ a 21 dias presença de hemossiderófagos).

Portanto, o padrão citológico é bastante útil na interpretação e formulação de diagnóstico diferencial, ou seja, a citologia pode indicar processos inflamatórios de etiologia alérgica, fúngica, bacteriana, parasitários entre outras e até processos físicos, como no caso da 
Avaliação clínica e citológica do trato respiratório de cavalos da raça quarto de milha, após o exercício

hemorragia induzida por exercício (EIPH). Segundo COWELL e TYLER (1992) é difícil mensurar valores de referência para o $B A L$, pois a contagem pode variar conforme a técnica de coleta e processamento. Porém, concordamos com os autores quando estes afirmam que a contagem diferencial de células no BAL de cavalos clinicamente normais mostra um predomínio de macrófagos e linfócitos (TABELA 1). Muitos desses trabalhos são realizados em cavalos de corrida e MAZAN e HOFFMAN (2003), referem em um estudo, significativa diferença entre o BAL de cavalos de corrida e cavalos de outros esportes, tais como tambor e baliza. Neste trabalho os resultados são similares, ou seja, os cavalos da raça Quarto de Milha só apresentam algum grau de sangramento, visível ao endoscópio ou a olho nu, após exercício intenso. O sangramento pode ser detectado por endoscopia 30 a 60 minutos após o exercício, que é o tempo necessário para a drenagem do sangue, das vias aéreas mais profundas para as de grande calibre. No BAL desses cavalos pode-se encontrar hemossiderófagos até 21 dias após exercício intenso ou prova (MAZAN e HOFFMAN, 2003).

A citocentrífuga é um equipamento de grande valor, pois ao concentrar as células, forma um botão que favorece a visualização e identificação celular em suas diferentes colorações, conseguindo-se contar no mínimo 5 células por campo (MAZAN e HOFFMAN, 2003). Neste experimento, a citocentrífuga preservou a morfologia das células adequadamente, principalmente dos linfócitos, contrastando com o resultado obtido por LAPOINTE et al. (1994). HEWSON e VIEL (2002) citam um decréscimo da percentagem de linfócitos com o uso de citocentrífuga. As colorações utilizadas permitiram a adequada visualização de todos os tipos celulares. A coloração de Papanicolau foi indispensável para a interpretação citológica em lâminas que apresentaram grumos celulares, uma vez que fornece uma visualização das camadas de células (macrófagos). A coloração especial - Azul da Prússia - foi utilizada para confirmar animais com algum grau de hemorragia pulmonar, como sugerem HEWSON e VIEL (2002), identificando, com segurança, os hemossiderógafos.

A utilização de cavalos da raça Quarto de Milha foi de grande importância neste estudo, apesar da limitada amostragem, conseguindo-se estabelecer uma avaliação citológica de BAL para a raça, em provas de três tambores e seis balizas. O número de animais parece ser limitante nos estudos de BAL em eqüinos, provavelmente, devido a dificuldade em se coletar, acondicionar e processar as amostras adequadamente e com a mesma metodologia, bem como os custos operacionais do processo. Alguns estudos foram realizados utilizando-se menos de 10 animais (MARLIN et al., 2002).

Para a coleta do BAL destes animais foi utilizado somente lidocaína intratraqueal, que reduziu a freqüência e a intensidade da tosse, corroborando os achados de WESTERMANN et al. (2005), não sendo necessária a sedação intravenosa, como sugerem DOUCET e VIEL (2002).

Conclui-se que a prevalência da EIPH pode variar conforme o método utilizado para detectá-la. Ela é menor quando o diagnóstico é realizado por meios visuais e aumenta quando se utiliza a citologia.

TABELA 1 - LEITURA DIFERENCIAL E TOTAL DE LEUCÓCITOS DO LAVADO BRONCOALVEOLAR DE CAVALOS, APÓS A INFUSÃO DE 100ML DE SOLUÇÃO SALINA 0,9\%.

\begin{tabular}{|c|c|c|c|c|c|c|c|c|c|c|}
\hline Número & $\begin{array}{l}\text { Volume } \\
\text { recuperado } \\
(\mathrm{mL})\end{array}$ & 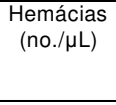 & 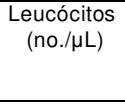 & $\begin{array}{c}\text { Macrófagos } \\
(\%)\end{array}$ & $\begin{array}{c}\text { Linfócitos } \\
(\%)\end{array}$ & $\begin{array}{c}\text { Neutrófilos } \\
(\%)\end{array}$ & $\begin{array}{c}\text { Eosinófilos } \\
(\%)\end{array}$ & $\begin{array}{c}\text { Mastócitos } \\
(\%)\end{array}$ & $\begin{array}{l}\text { Células } \\
\text { Epiteliais } \\
(\%)\end{array}$ & $\begin{array}{c}\text { Hemossi- } \\
\text { derófagos } \\
(\% \\
\text { macrófagos) }\end{array}$ \\
\hline 1 & 12 & NR & 300 & 79,7 & 14,5 & 0,5 & 0,0 & 4,1 & 1,2 & 25,0 \\
\hline 2 & 8 & NR & 350 & 82,4 & 6,4 & 0,0 & 0,0 & 0,0 & 11,2 & 0,5 \\
\hline 3 & 10 & NR & 200 & 67,0 & 32,8 & 0,2 & 0,0 & 0,0 & 0,0 & 0,5 \\
\hline 4 & 50 & NR & 450 & 71,3 & 28,7 & 0,0 & 0,0 & 0,0 & 0,0 & 76,0 \\
\hline 5 & 60 & NR & 385 & 67,7 & 29,1 & 0,9 & 0,0 & 2,3 & 0,0 & 15,0 \\
\hline 6 & 20 & 535 & 162 & 62,9 & 37,1 & 0,0 & 0,0 & 0,0 & 0,0 & 0 \\
\hline 7 & 25 & 315 & 460 & 69,9 & 28,7 & 1,5 & 0,0 & 0,0 & 0,0 & 1,0 \\
\hline 8 & 40 & 435 & 445 & 54,4 & 31,3 & 2,7 & 11,6 & 0,0 & 0,0 & 0,5 \\
\hline 9 & 38 & 312 & 442 & 57,7 & 42,1 & 0,2 & 0,0 & 0,0 & 0,0 & 19,0 \\
\hline 10 & 20 & 358 & 555 & 34,5 & 59,5 & 0,4 & 4,1 & 1,6 & 0,0 & 50,0 \\
\hline 11 & 42 & 228 & 162 & 48,4 & 49,7 & 0,2 & 0,4 & 1,3 & 0,0 & 40,0 \\
\hline 12 & 42 & 310 & 240 & 51,1 & 48,9 & 0,0 & 0,0 & 0,0 & 0,0 & 11,0 \\
\hline Média & 30,6 & 356,1 & 379,5 & 62,3 & 34,1 & 0,6 & 1,3 & 0,8 & 1,0 & \\
\hline Desvio & 17,0 & 100,3 & 131,8 & 13,7 & 14,8 & 0,8 & 3,4 & 1,3 & 3,2 & \\
\hline
\end{tabular}

$\mathrm{NR}=$ não realizado. 
QUADRO 1 - ACHADOS ENDOSCÓPICOS DA TRAQUÉIA E BRÔNQUIOS EM CAVALOS DA RAÇA QUARTO DE MILHA, APÓS TREINO PARA AS PROVAS DE SEIS BALIZAS E TRÊS TAMBORES.

\begin{tabular}{|c|c|c|c|c|c|c|}
\hline \multirow[b]{2}{*}{ Cavalos } & \multicolumn{4}{|c|}{ Secreção da Traquéia } & \multirow[b]{2}{*}{$\begin{array}{l}\text { Alteração na } \\
\text { Bifurcação da Traquéia }\end{array}$} & \multirow[b]{2}{*}{$\begin{array}{c}\text { Alteração nos } \\
\text { Brônquios principais }\end{array}$} \\
\hline & $\begin{array}{c}\text { Terço } \\
\text { Proximal }\end{array}$ & $\begin{array}{l}\text { Terço } \\
\text { Médio }\end{array}$ & $\begin{array}{l}\text { Terço } \\
\text { Distal }\end{array}$ & $\begin{array}{c}\text { Toda } \\
\text { Extensão }\end{array}$ & & \\
\hline 1 & & & $\mathrm{X}$ & & NDN & NDN \\
\hline 2 & $x$ & & & & NDN & NDN \\
\hline 3 & & & & $X$ & NDN & NDN \\
\hline 4 & & $x$ & & & NDN & NDN \\
\hline 5 & $X$ & & & & NDN & NDN \\
\hline 6 & & & & & NDN & NDN \\
\hline 7 & & $X$ & & & NDN & NDN \\
\hline 8 & $X$ & & & & NDN & NDN \\
\hline 9 & & & & & NDN & NDN \\
\hline 10 & & $X$ & & & NDN & NDN \\
\hline 11 & & & & & NDN & NDN \\
\hline 12 & & $X$ & & & NDN & NDN \\
\hline
\end{tabular}

$\mathrm{NDN}=$ Nada digno de nota

\section{Referências}

BACCARIN, R.Y.A. Diagnóstico e tratamento das pneumopatias de esforço. In: II Simpósio Internacional do Cavalo Atleta e IV Semana do Cavalo - SIMCAV. 2005, Belo Horizonte, Anais II Simpósio Internacional do Cavalo Atleta. Minas Gerais: Universidade Federal de Minas Gerais, 2005, p. 12-28.

CLARK, C.K.; LESTER, G.D.; VETRO, T.; RICE, B. Bronchoalveolar lavage in horses: effect of exercise and repeated sampling on cytology. Australian Veterinary Journal, v. 72, p 249-252, 1995.

COWELL, R.L.; TYLER, R.D. Cytology and hematology of the horse. Goleta: American Veterinary Publications, 1992.

DOUCET, M.Y.; VIEL, L. Clinical, radiographic, endoscopic, bronchoalveolar lavage and lung biopsy findings in horses with exercise-induced pulmonary hemorrhage.Canadian Veterinary Journal, v. 43, p 195202, 2002.

EPPINGER, M. Hemorragia pulmonar de esforço e o desempenho de eqüinos PSI \{Equus caballus\} em corridas de galope no Jockey Club do Paraná. 1990. Dissertação (Mestrado) - Universidade Federal do Paraná, Curitiba.

ERICKSON, H.H.; POOLE, D.C. Exercise-induced pulmonary hemorrhage. In: Equine Respiratory Disease. Ithaca, NY: International Veterinary Information Services. Disponível em: www.ivis.org. Accesso em 2003.

FORGARTY, U. Evaluation of a bronchoalveolar lavage tecnique. Equine Veterinary Journal, v. 3, p 174-176, 1990.

FORGARTY, U.; BUCKLEY, T. Bronchoalveolar lavage findings in horses with exercise intolerance. Equine Veterinary Journal, v. 6, p 434-437, 1991.
HEWSON, J.; VIEL, L Sampling, microbiology and citology of respiratory tract. In: Equine Respiratory Disease. Ithaca, NY: International Veterinary Information Services. Disponível em: www.ivis.org. Accesso em 2002.

KEEBLER, C.M.; SOMKAK, T.M. The Manual of Cytotechnology. Philadelphia: W.B. Saunders company, 1993.

LAPOINTE, J.M.; VRINS, A.; LAVOIC, J.P. Effects of centrifugation and specimen preparation technique on bronchoalveolar lavage analysis in horse. Equine Veterinary Journal, v.26. p 227-229,1994.

MCKANE, S.A.; SLOCOMBE, R.F. Sequential changes in bronchoalveolar cytology after autologous blood inoculation. Equine Veterinary Journal, Supplement, v. 30, p 126-130, 1999.

MARLIN, D.J.; SCHROTER, R.C.; CASHMAN, P.M.M.; DEATON, C.M.; POOLE, D.C.; KINDIG, C.A.; MCDONOUGH, P.; ERICKSON, H.H. Movements of thoracic and abdominal compartments during ventilation at rest and during exercise. In: VI International Conference on Equine Exercise Physiology. 2002, Kentucky, Equine Veterinary Journal, Supplement, v. 34. p 384-390, 2002.

MAZAN, M.R.; HOFFMAN, A.M. Clinical techniques for diagnosis of inflammatory airway disease in the horse. Clinical Tecniques in Equine Pratice, v. 2, p: 238 - 357, 2003.

MCGORUM, B.C.; DIXON P.M.; HALLIWELL, R.E.W.; IRVING, P. Comparison of cellular and molecular components of bronchoalveolarnlavage fluid harvested from different segments of the equine lung. Research in Veterinary Science, v. 55, p. 57-59, 1993.

OLIVER, O.; STAMPFLI, H.; AQUILERA, E. Enfermedades respiratórias de interés em el caballo de deporte. In: Conferencia Internacional de Caballos de deporte - CICADE. 2003, Curitiba. Anais Conferencia Internacional de Caballos de Deporte. Curitiba: Pontifícia Universidade Católica do Paraná, 2003, p. 15-22. 
Avaliação clínica e citológica do trato respiratório de cavalos da raça quarto de milha, após o exercício

ROY, M.F.; LAVOIE, J.P. Tool for the diagnosis of equine respiratory disorders. Veterinary Clinics of North America: equine practice, v. 19, p. 1-7, 2003.

SWEENEY, C.R.; BEECH, J. Bronchoalveolar Lavage. In: BEECH, J. Equine Respiratory Disorderas. Philadelphia: Lea \& Fabiger, 1991. p. $41-53$.
SWEENEY. C.R.; ROSSIER, Y.; ZIEMER, E.L. Effect of lung site and fluid volume on results of bronchoalveolar lavage fluid analysis in horses. American Journal of Veterinary Research, v. 53, p 1376-1379, 1992.

WESTERMANN, C.M.; LAAN, T.T.; VAN NIEWSTADT, R.A.; BULL, S.; FINK-GREMMELS, J. Effects of antitussive agents administered before bronchoalveolar lavage in horses American Journal of Veterinary Research, v.66, p 1420-1424, 2005.

Recebido para publicação: 12/02/2006 Aprovado:
$25 / 06 / 2006$ 